\title{
Fibromialgia: Revisión Sistémica
}

\section{Fibromyalgia: Systemic Review}

Dra. Dra. Mónica Solano Pochet. ${ }^{1}$

1 Médico general. Hospital clínica bíblica, San José Costa Rica.

Contacto: monicasolanopochet@gmail.com

\section{Resumen}

Fibromialgia (FM) se define como dolor musculoesquelético generalizado crónico usualmente acompañado por fatiga y alteraciones del sueño por mínimo tres meses que no se explican por otra condición médica. Es más común en mujeres adultas. La prevalencia es de $\mathbf{2 . 7 \%}$ en la población general. La etiología en sí es desconocida, pero se clasifica como una sensibilización central por cambios neuroendocrinológicos en sistema nervioso central y periférico en individuos genéticamente susceptibles, en respuesta a desencadenantes ambientales, resultando en percepción del dolor aumentada e hipersensibilidad a numerosos estímulos. Se caracteriza por dolor musculoesquelético generalizado y sensibilidad de tejidos blandos, acompañado por fatiga, alteraciones del sueño, trastornos somáticos, cognitivos y psiquiátricos. Pueden existir trastornos coexistentes que estimulan o exacerban la sintomatología. El diagnóstico se basa en la historia clínica y síntomas, examen físico normal, estudios complementarios normales y criterios AAPT o 2010 CAR positivos. Se deben valorar posibles diagnósticos diferenciales. El manejo se basa principalmente en las recomendaciones EULAR 2017 a través de medidas no farmacológicas y farmacológicas. Los pacientes tienen un deterioro en la calidad de vida y morbilidad, sin un aumento significativo en la mortalidad.

Palabras clave:

Fibromialgia, dolor crónico, dolor musculoesquelético generalizado crónico, sensibilidad, fatiga, alteraciones del sueño.

\section{Abstract}

Fibromyalgia (FM) is defined as chronic widespread musculoskeletal pain usually accompanied by fatigue and sleep disturbances of at least 3 months that are not explained by another medical condition. It is more common in women. The prevalence is $2.7 \%$ in the
Recibido: $10 /$ jul/2020

Aceptado: 18/nov/2020

Publicado: 15/dic/2020 general population. The etiology itself is unknown, but it is classified as a central sensitization by neuroendocrinological changes in the central and peripheral nervous system in genetically susceptible individuals, in response to environmental triggers, resulting in increased pain perception and hypersensitivity to numerous stimuli. It is characterized by generalized musculoskeletal pain and soft tissue tenderness, accompanied by fatigue, sleep disturbances, somatic, cognitive and psychiatric disorders. There might be coexisting disorders that stimulate or exacerbate symptoms. The diagnosis is based upon the clinical history and symptoms, normal physical examination, normal complementary studies and positive AAPT or 2010 RAC criteria. Possible differential diagnoses should be evaluated. Management is based on EULAR 2017 recommendations through non farmacological and farmacological measures. Patients have life quality deterioration and morbidity, without a significant increase in mortality.

Keywords:

Fibromyalgia, chronic pain, chronic widespread musculoskeletal pain, tenderness, fatigue, sleep disturbances. 


\section{Introducción}

La fibromialgia (FM) pertenece al grupo de trastornos reumáticos de tejidos blandos (1) y es la causa más común de dolor musculoesquelético generalizado crónico usualmente acompañado por fatiga, alteraciones cognitivas, síntomas psiquiátricos y somáticos $(1,2,3,4,8,10,12,13,14,15)$. El Colegio Americano de Reumatología (CAR) la define como un problema de salud común que causa dolor generalizado y sensibilidad que tiende a ir y venir en todo el cuerpo (1). A pesar de la presencia de dolor en tejidos blandos afectando los músculos, ligamentos y tendones $(2,3)$, no se asocia a inflamación en los tejidos y el dolor no se explica por otro trastorno reumático o sistémico (3). Usualmente se asocia a otras condiciones que pueden causar dolor musculoesquelético como alteraciones del sueño y síntomas psiquiátricos que deben ser considerados $(2,3)$. No hay anormalidades en el examen físico y los laboratorios y estudios radiológicos son normales (2). La etiología y fisiopatología son desconocidas $(2,5)$ y ha sido una condición controversial, ya que el rol orgánico de la enfermedad ha sido cuestionado y se ha considerado psicogénica o psicosomática, pero estudios recientes sugieren que es un trastorno en la regulación del dolor, clasificado como una sensibilización central $(2,12,13,14,15)$. Múltiples estresores físicos o emocionales pueden desencadenar o agravar los síntomas $(5,10,12)$. Es difícil realizar el diagnóstico y tiende a subdiagnosticarse. Llegar al diagnóstico puede tardar $>2$ años con los pacientes viendo un promedio de 3.7 médicos distintos en ese periodo $(8,9,10)$. El manejo está dirigido a reducir la mayoría de síntomas, puede ser difícil de tratar y por lo general responden a programas multidisciplinarios e individualizados $(4,6,12,13,15)$.

\section{Metodología}

Para la creación de este artículo, se consultaron bases de datos de UpToDate, PubMed, Clinical Key, The Cochrane para buscar artículos recientes sobre el tema en revisión. De los artículos encontrados, se seleccionaron 15 referencias bibliográficas. Los criterios de inclusión utilizados para la búsqueda fueron: A) Periodo de tiempo comprendido entre 2015 a 2020. B) Idioma inglés y español. Los criterios de exclusión fueron: A) Artículos publicados con más de 5 años de antigüedad, ya que se trata de una actualización en el tema. Para esto, se utilizaron términos de búsqueda como: "Fibromyalgia" combinando con "Clinical features", "Diagnosis", "Treatment", y "Management".

\section{Epidemiología}

FM es una causa común de dolor crónico y la causa más común de dolor musculoesquelético generalizado en mujeres de 20 a 55 años (2), es más común en mujeres $(1,2)$. Ocurre con mayor frecuencia en individuos ambiciosos, ocupados, con estrés constante y desempleados $(1,9)$. La prevalencia es de $2.7 \%$ (4.2\% mujeres y $1.4 \%$ hombres) $(10,13)$, incrementa con la edad (2) y nivel de educación (1).

\section{Etiología}

Aunque la etiología sigue siendo desconocida $(1,7,10,14)$, se considera un trastorno en la regulación del dolor, clasificado como sensibilización central. Una hipótesis bastante aceptada es que es un trastorno en la regulación del dolor por cambios neuroendocrinológicos en sistema nervioso central y periférico, que ocurre en individuos genéticamente susceptibles, posiblemente en respuesta a desencadenantes ambientales, resultando en percepción del dolor aumentada e hipersensibilidad a numerosos estímulos $(10,14)$. A continuación, se explican con mayor detalle los descubrimientos recientes sobre los mecanismos involucrados en la FM:

1. Alteración del procesamiento del dolor en el Sistema Nervioso Central: La evidencia ha demostrado que es un trastorno en el procesamiento del dolor, lo cual incluye: una sumatoria temporal del dolor ya que experimentan mayores incrementos en la percepción de la intensidad del dolor cuando estímulos nocivos son administrados, disminución endógena en la inhibición del dolor porque los sistemas endógenos de analgesia parecen estar deficientes al haber reducción en el control inhibitorio de estímulos nocivos e incapacidad para inhibir estímulos sensoriales irrelevantes, alteración en los receptores del dolor y los neuropéptidos relacionados al dolor por; cambios en los receptores de opioides que incluyen mayor regulación en la periferia y reducción en el cerebro, aumento de sustancia $\mathrm{P}$ (neuropéptido asociado a estados de dolor crónico) en el líquido cefalorraquídeo e incremento del factor neurotrófico derivado del cerebro en plasma y cerebro. Finalmente, los estudios de neuroimagen han demostrado disregulación del dolor basados en los siguientes aspectos $(5,9)$ :

a) Respuesta al dolor aumentada: Por medio de imágenes de resonancia magnética (IRM) funcional se ha demostrado que tienen mayor actividad neuronal en las regiones cerebrales de procesamiento del dolor como la corteza somatosensorial secundaria, la ínsula y la corteza cingulada anterior. Durante ensayos del dolor por IRM presentan menor impacto en la 
reducción del dolor bajo contextos emocionales positivos y presentan mayor actividad neuronal con catástrofes $(5,9,10)$. b) Cambios en la morfología cerebral: Los pacientes muestran por IRM una reducción significativa del volumen total de materia gris y un mayor aumento de pérdida de materia gris asociada a la edad, lo cual sugiere un envejecimiento cerebral prematuro. Esta pérdida, fue más prominente en las regiones relacionadas al procesamiento del estrés y del dolor. Una revisión sistemática de estudios por imagen encontró evidencia moderada que la sensibilización central está correlacionada con la disminución de materia gris en regiones cerebrales específicas (5).

c) Alteración en la función de neurotransmisores: Utilizando IRM con espectroscopia de protones los pacientes tenían niveles más altos de glutamina dentro de la ínsula posterior derecha, los niveles más altos de glutamato se asociaron con umbrales de dolor por presión más bajos. Datos más limitados, utilizando tomografía por emisión de positrones, han mostrado una actividad dopaminérgica reducida en la respuesta al dolor. Se evaluó la excitabilidad cortical y la modulación intracortical mediante estimulación magnética transcraneal de la corteza motora, observándose déficits en la modulación intracortical de los mecanismos GABAérgicos y glutamaérgicos. Utilizando espectroscopia de RM, mostraron niveles más altos de glutamato y una relación glutamina-glutamato/creatina más alta en la amígdala derecha. En los pacientes con más dolor, fatiga y síntomas depresivos, los niveles de inositol eran más altos en la amígdala derecha y el tálamo derecho. Los niveles de GABA en la ínsula anterior derecha fueron más bajos. Dentro de la ínsula posterior derecha, los niveles más altos de GABA se correlacionaron con los umbrales de presión-dolor (5).

d) Cambios en la actividad cerebral funcional en estado de reposo: En un estudio con IRM funcional se observó una conectividad funcional anormal en estado de reposo del gris periacueductal, sugiriendo que los cambios resultan en un deterioro de la inhibición del dolor descendente. Se observó una conectividad funcional alterada con la red en modo predeterminado (una región activa cuando el cerebro está en reposo) y la ínsula, una región clave para el procesamiento del dolor. En estado de reposo demostró una estructura central alterada, regiones que transmiten información neuronal de manera eficaz. La topología central alterada dentro de la ínsula se asoció con la intensidad del dolor clínico. Se utilizó IRM Funcional para identificar una señal cerebral que puede caracterizar la FM siguiendo las respuestas a la presión dolorosa y los estímulos multisensoriales no dolorosos. Los patrones multisensoriales específicos relacionados con el dolor clasificaron a los pacientes con FM frente a los controles con una sensibilidad del $92 \%$ y especificidad del $94 \%$. Los autores sugirieron que tales señales cerebrales podrían resultar en dianas neurológicas objetivas para la terapia (5).

2. Estrés y Disfunción del sistema nervioso autonómico (SNA): La asociación del dolor con alteraciones del sueño, hu mor y cognitivas se vincula a la reactividad del estrés y a la disfunción del sistema nervioso autónomo. Las alteraciones del sueño aumentan el dolor, incrementando la reactividad simpática cardiovascular (5).

a) La hiperactividad en respuesta al estrés ha sido demostrada por anormalidades en el eje hipotalámico pituitario adrenal (EHPA) a través de distintos estudios realizados. Hubo una correlación entre los niveles cerebroespinales del factor liberador de corticotropina, el dolor sensorial y la variación de la función autónoma. También hubo una fuerte correlación entre los niveles de cortisol y el dolor al despertar y una hora después del despertar. Los sujetos con dolor crónico generalizado tenían niveles de cortisol sérico más altos y hubo una correlación significativa entre la disfunción del EHPA y el desarrollo de dolor crónico generalizado (5).

b) La evidencia de disfunción del SNA incluye: Se demostró una disminución de la capacidad de respuesta a la esti mulación beta adrenérgica mediante pruebas in vitro de generación de AMP cíclico mediada por receptores beta adrenérgicos. En un estudio se evaluaron las catecolaminas urinarias y la frecuencia cardíaca durante un perío do de 24 horas en un entorno hospitalario controlado (incluida la relajación, una prueba con estrés mental prolongado y el sueño) y durante la actividad diaria. Los niveles de catecolaminas fueron menores. Tenían niveles de adrenalina más bajos durante la noche y el segundo día y tenían niveles de dopamina más bajos durante el primer día, la noche y el segundo día. En general, la frecuencia cardíaca fue mayor. Los índices de variabilidad de la frecuencia cardíaca nocturna fueron diferentes en mujeres con FM, estos parámetros se correla cionaron con varios síntomas, incluida la gravedad del dolor. Los pacientes suelen mostrar una respuesta al estrés hipertónico, que incluye un aumento de la presión arterial, cuando se exponen a un estímulo doloroso (5).

3. Predisposición genética: estudios observacionales y biológicos sugieren que el dolor crónico generalizado y la FM tienen en parte una base genética $(5,9)$, ya que hay mayor incidencia de FM en familiares de primer grado de los pacientes (14). Sin embargo, no se ha logrado establecer una asociación con un gen específico (5).

4. Anormalidades cognitivas, humorales y del sueño: La disfunción del sistema nervioso central subyacente es sugeri da por las alteraciones del sueño, humor y cognitivas. La actividad del sueño en fase alfa es la más característica. 


\section{CIENCIA\&SALUD}

Las alteraciones cognitivas, humorales y del sueño están interconectadas y se correlacionan con la severidad (5).

5. Mecanismos periféricos de dolor: Los generadores de dolor periférico incluyen puntos gatillo miofasciales o liga mentosos, u osteoartritis de las articulaciones y columna $(5,12)$.

6. Anormalidades inmunes: Hay poca evidencia que apoye un trastorno inmunológico $(5,9)$.

\section{Manifestaciones clínicas}

FM se caracteriza por dolor musculoesquelético generalizado, acompañado por otros síntomas somáticos, particularmente fatiga y alteraciones del sueño, trastornos cognitivos y psiquiátricos $(1,2,14)$. Al examen físico hay sensibilidad en múltiples puntos de tejidos blandos $(2,14)$. Algunos trastornos se manifiestan con mayor frecuencia y ciertas características de estos pueden estimular o exacerbar los síntomas (2). Los síntomas principales son dolor generalizado, fatiga y alteraciones del sueño por mínimo 3 meses y no se explican por otra condición médica $(2,14)$.

- Dolor musculoesquelético generalizado: Es el síntoma principal $(1,2,9,14)$, por lo general mínimo 6 puntos de dolor están involucrados que pueden incluir la cabeza, brazos, piernas, abdomen, espalda, columna, trasero. En ocasiones, inicialmente puede ser localizado en cuello y hombros. No necesariamente todas las partes del cuerpo están afectadas simultáneamente, pero es importante reconocer que no es un fenómeno localizado. Los pacientes suelen referir que "les duele por todo lado" $(2,14)$. Usualmente el dolor es constante y crónico, variando intensidad. Tienen puntos de sensibilidad $(1,14)$ en áreas del cuerpo sensibles a la presión y por lo general, no se han percatado de la presencia de esos puntos como dolorosos hasta la examinación (14).

- Fatiga y alteración del sueño: Fatiga persistente y alteraciones del sueño son manifestaciones principales. Actividades menores o inactividad prolongada pueden aumentar los síntomas. Amanecen con rigidez y cansados, aunque hayan dormido 8-10 horas. Característicamente duermen muy ligero, despiertan frecuentemente, tienen dificultad para conciliar el sueño $(2,14)$ y no es restaurador $(1,2,14)$.

- Alteraciones cognitivas: describen problemas para poner atención y hacer tareas que requieran un cambio rápido de pensamiento $(1,2,9)$.

- Síntomas psiquiátricos: Al momento del diagnóstico 30-50\% presenta depresión o ansiedad, trastornos del humor y comportamiento $(2,14)$. Los trastornos de ansiedad, trastorno bipolar, síndrome de estrés postraumático y rasgos catastrófico o alexitimia son más frecuentes (2).

- Cefalea: presente en $>50 \%$ de los pacientes (2). FM es común en pacientes con migraña (2).

- Parestesias: con frecuencia refieren adormecimiento, hormigueo, sensación quemante o que algo les camina (2).

- Otros síntomas y trastornos: como dolor abdominal o en tórax, síntomas sugestivos de síndrome de intestino irritable, dolor pélvico, síntomas urinarios, síntomas de disfunción del sistema nervioso autonómico, ojos secos, fenómeno de Raynaud, hipotensión ortostática, frecuencia cardiaca alterada y pérdida de audición $(1,2)$.

- Impacto psicosocial: Muchos pacientes sufren FM por meses e inclusive años antes del diagnóstico, interrumpiendo la vida diaria en funcionamiento y calidad (14).

- Trastornos coexistentes: Algunos grupos de trastornos pueden ocurrir en asociación y ciertas características de estas condiciones pueden estimular o exacerbar los síntomas. El reconocimiento y tratamiento efectivo para estas comorbilidades puede aliviar potencialmente los síntomas de FM (2). Estas condiciones incluyen: Síndromes somáticos funcionales y trastornos relacionados: síndrome de intestino irritable, síndrome de fatiga crónica, migraña y cefalea tensional, síndromes de dolor de vejiga y pélvico crónico, trastornos temporomandibulares. Trastornos psiquiátricos: trastornos depresivos y ansiosos, y síndrome de estrés postraumático. Alteraciones del sueño: apnea de sueño, síndrome de piernas inquietas, trastornos periódicos del movimiento de caderas $(2,3)$. Enfermedades reumáticas inflamatorias: La prevalencia de FM está aumentada en pacientes con artritis inflamatoria crónica, enfermedades reumáticas autoinmunes sistémicas como Artritis reumatoide, artritis psoriática, espondiloartritis, lupus eritematoso sistémico, síndrome de Sjogren, osteoartritis y trastornos regionales de dolor (2). Trastornos ginecológicos como endometriosis y vulvodinia (14).

\section{Diagnóstico}

FM se debe sospechar en pacientes con dolor crónico por al menos 3 meses de duración $(2,3,9,14)$ sin otras causas identificadas. Se basa en los síntomas, principalmente dolor musculoesquelético generalizado acompañado por problemas moderados o severos del sueño y fatiga. Aunque la sensibilidad puede estar presente en múltiples sitios, hay ausencia de inflamación articular u otros cambios inflamatorios $(2,14)$. No hay exámenes complementarios o biomarcadores para el 
diagnóstico, por lo que es meramente clínico $(2,7)$. El diagnóstico temprano mejora la satisfacción con la salud y reducción de la utilización de recursos y costos asociados (10). La evaluación diagnóstica incluye historia y examen físico, con laboratorios limitados para excluir otras condiciones $(2,14)$.

Historia Clínica: Características del dolor: localización, duración, calidad y severidad (1,2). Se recomiendan 2 instrumentos para su evaluación; Criterios AAPT (ACTTION-APS Taxonomía del Dolor) de la ACTTION, FDA y Asociación Americana del dolor o los criterios del CAR. Trastornos del sueño, fatiga y otros síntomas: Se debe preguntar sobre el sueño, energía mental y física, alteraciones cognitivas, trastornos del humor y otras condiciones psiquiátricas. Síntomas de migraña crónica o cefaleas, síndrome de intestino irritable, dolor pélvico crónico o de vejiga o dolor temporomandibular crónico. Otros trastornos y diagnósticos diferenciales: historia de condiciones que pueden causar dolor musculoesquelético que puede coexistir o simular FM (2).

Examen Físico: Se debe hacer un examen físico amplio, con atención especial a la examinación articular y neurológica para identificar dolor generalizado y sensibilidad de tejidos blandos y excluir otros diagnósticos. Se debe palpar sitios articulares y de tejidos blandos, y examinar por sinovitis. Ya no se recomienda palpar sitios específicos de sensibilidad o enumerar el número de estos, sino estimar la diseminación y localización múltiple de sensibilidad de tejidos blandos, estos sitios son los puntos de criterios de clasificación del CAR y se localizan en mitad superior del trapecio, epicóndilo lateral, segunda unión costocondral, trocánter mayor y otras locaciones y suelen ser un hallazgo físico que casi siempre está presente. Los pacientes no tienen tanta sensibilidad en las articulaciones, no causa edema o eritema. La evaluación neurológica a veces revela anormalidades motoras o sensitivas menores en ausencia de otra enfermedad (2).

Laboratorios y otros estudios: FM no causa alteraciones en exámenes de laboratorio o de imagen, se pueden realizar ciertos exámenes para descartarse otro diagnóstico $(1,2,10,14)$. Normalmente se obtiene un hemograma completo, velocidad de eritrosedimentación y proteína $\mathrm{C}$ reactiva que suelen estar normales y descartan un proceso inflamatorio oculto. Los exámenes serológicos como anticuerpos antinucleares y factor reumatoide deben realizarse si hay historia o examen físico que sugiera una enfermedad inflamatoria, sistémica o reumatológica $(2,14)$. En pacientes con sospechas de enfermedad tiroidea o inflamatoria muscular se envían pruebas de función tiroidea o creatinina quinasa $(1,2,10,14)$.

Clasificación y criterios diagnósticos: Se recomienda utilizar los criterios del 2010 del CAR o los criterios AAPT (2). Sin embargo, las guías no reemplazan la impresión clínica y el diagnóstico basado en síntomas (2). Anteriormente se utilizaban los Criterios del CAR de 1990 (2), actualmente se sugiere que los puntos sensibles de examinación no deben usarse para el diagnóstico $(1,2,7)$ y que la presencia de síntomas somáticos, como alteraciones del sueño y fatiga, se deben incluir en los criterios $(2,7)$.

Criterios AAPT: En el 2013 la ACTTION se asoció con la FDA y la Asociación Americana del Dolor y crearon el AAPT como sistema diagnóstico (2). Los criterios que utiliza son: dolor generalizado en 6 o más lugares dolorosos de un total de 9 posibles sitios, alteraciones del sueño o fatiga, presentes por al menos 3 meses $(2,9)$. La presencia de otro trastorno doloroso o síntomas relacionados no excluyen el diagnóstico (2). En la taxonomía, se incluyen 5 dimensiones; Dimensión 1 Criterios diagnósticos principales, Dimensión 2 Características comunes, Dimensión 3 Comorbilidades médicas comunes, Dimensión 4 Consecuencias neurobiológicas, psicosociales y funcionales, Dimensión 5 Mecanismos neurobiológicos y psicosociales, factores de riesgo y protectores $(7,9)$.

2010 CAR Criterios diagnósticos preliminares: No requiere la examinación de puntos de sensibilidad, tiene una escala que mide la severidad de los síntomas característicos $(2,10)$. En el 2011 se modificó y se agregó una página de autoevaluación de los síntomas por parte del paciente (10). Un paciente cumple los criterios diagnósticos si cumple las 3 condiciones: Índice de dolor generalizados $>7$ y severidad de síntomas $>5$ o Índice de dolor generalizados 3-6 y severidad sintomática $>9$, síntomas presentes por mínimo de 3 meses, no hay otro trastorno que puede explicar los síntomas. El índice de dolor generalizado es una medida del número de regiones corporales dolorosas de una lista de 19 áreas. El índice de severidad sintomática incluye un estimado del grado de fatiga, despertares cansados, síntomas cognitivos y síntomas somáticos en general (2). 


\section{CIENCIA\&SALUD}

\section{Diagnósticos diferenciales}

Los múltiples síntomas inespecíficos de FM pueden simular otras condiciones y la consideración de diagnósticos diferenciales es importante (2).

- Artritis y enfermedades reumatológicas sistémicas: Artritis reumatoide, Síndrome de Sjogren, Lupus eritematoso sistémico, Spondyloartritis, Polimialgia reumática, osteoartritis $(3,9)$

- Enfermedades musculares y mialgia: Miopatías inflamatorias y metabólicas, Miopatía por estatinas, mialgia (3).

- Trastornos infecciosos, metabólicos y neurológicos: infección por virus como hepatitis, chikungunya, VIH, VLTH, enfermedad de Lyme. Endocrinopatías como hipotiroidismo, hiperparatiroidismo, síndrome de Cushing, enfermedad de Addison, insuficiencia adrenal y déficit de vitamina D. Trastornos neurológicos como neuropatías periféricas, síndrome del túnel carpal, esclerosis múltiple, miastenia gravis, neuropatía de fibras pequeñas e intolerancia ortostática $(3,9)$.

- Dolor regional de tejidos blandos: síndrome de dolor miofascial, tendinitis y bursitis (3).

\section{Manejo}

Según las guías de recomendaciones EULAR 2017 el manejo requiere un diagnóstico pronto, brindándole al paciente información con material escrito sobre su condición y educándole sobre esta. Debe haber una evaluación exhaustiva del dolor, funcionalidad y contexto psicosocial. El manejo debe ser realizado de forma gradual con el objetivo de mejorar la calidad de vida. Debe enfocarse primeramente en las modalidades no farmacológicas basado en la disponibilidad, costo, seguridad y preferencias del paciente, en caso de no presentar mejoría con estas, se recomienda proceder con las farmacológicas individualizadas según las necesidades del paciente. Las recomendaciones son las siguientes $(4,11)$ :

1. Historia y examen físico, diagnóstico de fibromialgia (si fuese necesario para excluir comorbilidades o diganóstico diferenciales realizar: laboratorios y/o exámenes radiológicos y referir a especialistas) (11).

2. Educación al paciente: Se debe reafirmar que es una enfermedad real y de naturaleza benigna, usualmente no progresiva $(4,15)$. Explicar que es una alteración del dolor $(4,15)$, que los síntomas van a ir y venir, pero que por lo general cierto dolor y fatiga persisten, aunque la mayoría logra vivir una vida normal y activa. Realizar una revisión del rol del estrés y alteración del humor para alentar al paciente a aprender técnicas de relajación. Educar en hi giene del sueño y el beneficio potencial de corregir malos hábitos, reconocer y obtener tratamiento para estas al teraciones. Aconsejar sobre la importancia del ejercicio para acondicionamiento y mejorar la capacidad funcional $(4,10)$.

3. Ejercicio físico: se considera una recomendación fuerte, por su efecto beneficioso sobre el dolor, función física y bienestar, disponibilidad, bajo costo y seguridad $(4,11)$. Se debe escoger individualizado en la preferencia y estado físico del paciente (4). Las actividades aeróbicas de bajo impacto como caminar rápido, ciclismo, natación y aeróbi cos acuáticos son las intervenciones más exitosas $(4,8,15)$.

Las terapias de movimientos meditativos o de concientización, se consideran una recomendación débil, pero ayu dan a mejorar el sueño, fatiga y calidad del sueño (11).

La acupuntura e hidroterapia se consideran una recomendación débil, ayudan a mejorar el dolor, la fatiga y la cali dad de vida (11).

Terapia Física: puede ayudar a reducir el dolor periférico que puede actuar como desencadenante $(6,11)$.

4. Si estas recomendaciones no funcionan se recomiendan terapias individualizadas basadas en las necesidades del paciente (11) e identificar comorbilidades. Explicar las comorbilidades como un componente interrelacionado y que es probable que respondan al plan de tratamiento general (4). Como las siguientes:

a) Depresión relacionada al dolor, ansiedad, pensamiento catastrófico: Terapias psicológicas son una reco mendación débil, pero deben ser consideradas en pacientes con trastornos del estado de ánimo (11), como terapia cognitivo conductual $(1,6,7,11,15)$ o para trastornos más severos como depresión y ansie dad se pueden considerar tratamientos psicofarmacológicos (11).

b) Dolor severo o alteraciones del sueño: se deben considerar teraías farmacológicas en pacientes con dolor severo (duloxetina, pregabalina, tramadol o combinación con paracetamol) o con alteraciones del sueño (dosis bajas de amitriptilina, ciclobenzaprina, pregabalina en la noche) (11). Se sugiere iniciar con antidepresivos tricíclicos y drogas relacionadas a dosis bajas por las noches $(4,7,15)$. Usualmente se inicia con Amitriptilina 5-10mg 1-3 horas antes de dormir $(4,15)$, la dosis se puede incrementar $5 \mathrm{mg}$ cada 2 se manas en caso necesario, sin exceder $75 \mathrm{mg}$. Ciclobenzaprina se inicia con dosis de $5-10 \mathrm{mg}$ antes de dor mir y se incrementa según la tolerancia hasta $30-40 \mathrm{mg}$ (4). Pregabalina se inicia $25-50 \mathrm{mg}$ antes de dormir aumentando la dosis según requerimientos hasta $300-450 \mathrm{mg} /$ día $(4,10)$. Gabapentina se usa como alter nativa a pregabalina, se inicia $100 \mathrm{mg}$ antes de dormir con incrementos hasta $1200-2400 \mathrm{mg} /$ día, usual 


\section{CIENCIA\&SALUD}

mente divididas en 3 tomas (4).

c) En los que no responden a monoterapia, se puede dar terapia combinada con fármacos de distintas cla ses o referir a reumatología o psiquiatría según las comorbilidades (6).

d) Discapacidad severa: se recomiendan programas de rehabilitación multimodal son una recomendación débil, tienen mejores resultados que las terapias individuales (11).

e) Respuesta inadecuada: Los pacientes con respuestas inadecuadas o con síntomas a pesar de las medidas no farmacológicas y farmacológicas a las dosis máximas toleradas pueden requerir cambiar a medicamen tos alternativos. Se debe evaluar la adherencia al tratamiento y el manejo de comorbilidades, ya que esto podría interferir con la eficacia del tratamiento $(4,6)$. Por lo general, se prueba un fármaco de cada cla se on aumentos de dosis por 3 meses antes de realizar un cambio. La selección de otro tratamiento se hace basada en los síntomas prominentes del paciente (4).

5. No se recomiendan: terapia de biorretroalimentación, hipnoterapia, capsaicina y otras terapias alternativas por su falta de efectividad. No se recomiendan terapias quiroprácticas por falta de seguridad (11). No se recomienda el uso de AINEs, IMAOs, ISRS por falta de eficacia $(1,4,6,7,8,11,15)$. Fuertemente no se recomienda el uso de hor mona de crecimiento, oxibato de sodio, opioides de alta potencia y corticoesteroides por su falta de eficacia y alto riesgo de efectos secundarios (11).

\section{Atención Primaria}

FM es un diagnóstico difícil para muchos médicos de atención primaria y se suelen referir a reumatología (2,10). Aunque el entendimiento ha aumentado en los últimos años, las barreras para el diagnóstico y el tratamiento óptimo son múltiples y variadas (10).

- Impedimentos para establecer el diagnóstico: No hay anormalidades objetivas físicas, de laboratorio o imagen y el diagnóstico se baja en el reporte de síntomas subjetivos (2). A pesar de las mejoras en el entendimiento de la condición, se sigue subdiagnosticando y subtratando. Una gran cantidad de médicos, principalmente de atención primaria reportan criterios diagnósticos no claros, entrenamiento y habilidad insuficiente para diagnosticar FM y falta de conocimiento de las opciones de tratamiento (10).

- Beneficios de establecer el diagnóstico: El reconocimiento y diagnóstico de FM lleva a disminución del uso de recursos, incluyendo exámenes y costos de salud (2).

- Estrategias para manejar FM en atención primaria: La clave es un acercamiento integral, con apoyo clínico y no clínico, educación multifacética, claridad de los objetivos y expectativas. Educación médica para tener las herramientas y entrenamiento para reconocer los síntomas y tener la confianza de prescribir los tratamientos. La educación al paciente es crítica para ayudar al paciente a entender, aceptar y manejar su condición. Poner metas para el tratamiento es importante para que el paciente conozca las limitaciones de los tratamientos actuales. Utilizar un tratamiento multimodal integrado con tratamiento farmacológico y no farmacológico, necesitando de un equipo multidisciplinario para las comorbilidades. Se debe dar seguimiento del proceso y monitorización de síntomas para evaluar el impacto y efectividad del tratamiento (10).

\section{Pronóstico}

FM tiene un deterioro significativo en la calidad de vida (15). La mayoría continúan teniendo dolor crónico y fatiga, y experimentan mayor discapacidad para el trabajo. Los factores demográficos y psicosociales impactan el pronóstico y el resultado, la depresión, historias de abusos, preocupación somática excesiva y el pensamiento catastrófico son los factores más importantes para un resultado adverso. También, el género femenino, estado socioeconómico bajo y el desempleo se asocian a efectos adversos. La obesidad y un índice de masa corporal elevado se asocia a incremento de síntomas, mayor dolor y disminución de la calidad de vida. La participación en un trabajo productivo se correlaciona con mejor funcionamiento cognitivo y físico (4). Las alteraciones del sueño parecen ser el síntoma más persistente a pesar del tratamiento (9). Los pacientes con síntomas severos experimentan mayor morbilidad, comorbilidades y un incremento en los costos de tratamiento. En general, los índices de mortalidad no parecen estar aumentados, pero si se puede presentar un aumento en el riesgo de suicidio $(4,9)$. El compromiso de atención médica ambulatoria es un factor protector contra el suicidio (4).

\section{Conclusiones}

FM es la causa más común de dolor musculoesquelético generalizado crónico usualmente acompañado por fatiga y alteraciones del sueño, por mínimo 3 meses de duración que no se explican por otra condición médica. La etiología en sí es desconocida, pero se clasifica como una sensibilización central con trastorno en la regulación del dolor. Es más común en mujeres 


\section{CIENCIA\&SALUD}

adultas. El diagnóstico se basa en los síntomas de la historia clínica, examen físico y criterios de evaluación, no hay laboratorios o exámenes complementarios que confirmen el diagnóstico. Existen diagnósticos diferenciales que deben tomarse en cuenta a la hora de realizar el diagnóstico. Pueden haber trastornos coexistentes con FM. El tratamiento es dirigido a reducir la mayoría de síntomas y se basa en tratamiento no farmacológico como educación, identificación de comorbilidades, ejercicio, terapia física, terapia psicológica, y farmacológica. Conlleva un deterioro en la calidad de vida y en la morbilidad, pero los índices de mortalidad no tienden a aumentarse significativamente. Se debe intentar educar a los profesionales de la salud sobre el diagnóstico y manejo de los pacientes con FM para evitar el subdiagnóstico y poder abordarlos correcta y tempranamente, mejorando así la calidad de vida del paciente y reduciendo costos de salud.

\section{Bibliografía}

(1) Anna Binkiewicz-Glinska, Stanislaw Bakula, Hanna Tomczak, Jerzy Landowski, Katarzyna Ruckemann-Dziurdzinska, Katarzyna Zaborowska-Sapeta et al. Fibromyalgia Syndrome - a multidisciplinary approach. Psychiatr Pol. 2015; 49 (4): 801-810. Recuperado de: DOI: $h t t p: / / d x . d o i . o r g / 10.12740 / p s y c h i a t r i a p o l s k a . p l / o n l i n e-f i r s t / 4$

(2) Don L Goldenberg, MD. Clinical manifestations and diagnosis of fibromyalgia in adults. UpToDate. 2019 Nov 7: 1-33. Recuperado de: https://www.uptodate.com/contents/clinical-manifestations-and-diagno...search_result\&selectedTitle $=1 \sim 150 \&$ usage_type=default\&display_rank=1

(3) Don L Goldenberg, MD. Differential diagnosis of fibromyalgia. UpToDate. $2018 \mathrm{Jul}$ 27: 1-25. Recuperado de: https://www. uptodate.com/contents/differential-diagnosis-of-fibromya...search_result\&selectedTitle=5 $150 \& u s a g e \_t y p e=d e f a u l t \& d i s-$ play_rank=5

(4) Don L Goldenberg, MD. Initial treatment of fibromyalgia in adults. UpToDate. 2020 Jan 23: 1-41. Recuperado de: https:// www.uptodate.com/contents/initial-treatment-of-fibromyalgia-...=search_result\&selectedTitle=3 $150 \&$ usage_type=default\&display_rank=3

(5) Don L Goldenberg, MD. Pathogenesis of fibromyalgia. UpToDate. 2019 Sep 26: 1-23. Recuperado de: https://www.uptodate.com/contents/pathogenesis-of-fibromyalgia/prin...search_result\&selectedTitle=6 150\&usage_type=default\&display_ rlssuank $=6$

(6) Don L Goldenberg, MD. Treatment of fibromyalgia in adults not responsive to initial therapies. UpToDate. 2020 Jan 14: 1-35. Recuperado de: https://www.uptodate.com/contents/treatment-of-fibromyalgia-in-adul...search_result\&selectedTitle $=2 \sim 150 \&$ usage_type=default\&display_rank $=2$

(7) F Atzeni, R Talotta, IF Masala, C Giacomelli, C Conversano, V Nucera et al. One year in review 2019: fibromyalgia. Clin exp Rheumatol. 2019; 37 (116): S3-S10.

(8) G J Macfarlane, C Kronisch, L E Dean, F Atzeni, W Hauser, E FluB et al. EULAR revised recommendations for the management of fibromyalgia. Ann Rheum Dis. 2017; 76: 318-328. Recuperado de: doi:10.1136/annrheumdis-2016-209724

(9) Lesley M Arnold, Robert M Bennett, Leslie J Crofford, Linda E Dean, Daniel J Clauw, Don L Goldenberg et al. AAPT Diagnostic Criteria for Fibromyalgia. The Journal of Pain. 2019 June; 20 (6): 611-628. Recuperado de: https://doi.org/10.1016/j. jpain.2018.10.008

(10) L M Arnold, K B Gebke, E H S Choy. Fibromyalgia: management strategies for primary care providers. Int J Clin Pract. 2016 Feb; 70 (2): 99-112. Recuperado de: doi: 10.1111/ijcp.12757

(11) Macfarlane GJ, Kronisch C, Dean LE, Atzeni F, Hauser W et al. EULAR revised recommendations for the management of fibromyalgia. Ann Rheum Dis. 2017; 76: 318-328. Recuperado de: doi:10.1136/annrheumdis-2016-209724

(12) Robert M Bennett. Pain management in fibromyalgia. Pain Manag. 2016; 6 (4): 313, 316. Recuperado de: 10.2217/pmt2016-0026

(13) Thorpe J, Shum B, Moore RA, Wiffen PJ, Gilron I. Combination pharmacotherapy for the treatment of fibromyalgia in adults (Review). Cochrane Database of Systematic Reviews. 2018; Issue 2. Art No: Art. No.: CD010585: 1-63. Recuperado de: doi: 10.1002/14651858.CD010585.pub2.

(14) Yukiko Kimura, MD, Gary A Walco, PhD. Fibromyalgia in children and adolescents: Clinical manifestations and diagnosis. UpToDate. 2019 Mar 8: 1-19. Recuperado de: https://www.uptodate.com/contents/fibromyalgia-in-children-and-adol... search_result\&selectedTitle $=8 \sim 150 \&$ usage_type $=$ default\&display_rank $=8$

(15) Yukiko Kimura, MD, Gary A Walco, PhD. Fibromyalgia in children and adolescents: Treatment and prognosis. UpToDate. 2019 Mar 8: 1-14. Recuperado de: https://www.uptodate.com/contents/fibromyalgia-in-children-and-adol...search_result\&selectedTitle=7 150\&usage_type=default\&display_rank=7 